\title{
Development of the Japanese version of the AACN synergy model for patient care
}

\begin{abstract}
In this study, we translated the AACN Synergy Model for Patient Care into Japanese, and investigated the value of applying the synergy model to simulated case studies of acute patients.

The permission of the original model developers was obtained before work began on producing the Japanese version. Preliminary testing was carried out to verify the effects of using the Japanese version of the model. The participants in this study were 13 nurses with at least five years of nursing experience (mean 9.9 \pm 4.5 years) who were working in Intensive Care Unit (ICU) or High Care Unit (HCU) wards. The simulated case studies used in this research were based on those described in the utilization of the original synergy model.

By evaluating the case studies using the Japanese version of the synergy model, we could confirm that the order of importance assigned to items was slightly consistent between participants (Case 1: Patient characteristics: Kendall's $W=0.356, \chi^{2}=27.39, d f=7$; Nursing competencies: Kendall's $W=0.396, \chi^{2}=27.7, d f=7$ ) (Case 2: Patient characteristics: Kendall's $W=0.358, \chi^{2}=27.568, d f=7$; Nursing competencies: Kendall's $W=0.499, \chi^{2}=38.424, d f=7$ ). Therefore, our results suggested that the Japanese version of the synergy model could be applied to clinical practice in Japan. In the future, however, larger samples are required in order to truly test the validity and reliability of this new tool.
\end{abstract}

Keywords: Japanese version, aacn synergy model, simulated case study, patient-nurse relationship
Volume 2 Issue 5 - 2017

\author{
Yoshiaki Ito,' Kyoko Tokoro, ${ }^{2}$ Takayasu \\ Kawaguchi $^{3}$ \\ 'Department of Nursing, Tokyo University of Information \\ Sciences, Japan \\ ${ }^{2}$ Nursing Science, Graduate School of Comprehensive Human \\ Sciences, University of Tsukuba, Japan \\ ${ }^{3}$ Department of Nursing, Tokyo University of Information \\ Sciences, Japan
}

Correspondence: Yoshiaki Ito, Department of Nursing, Tokyo University of Information Sciences, Chiba-city, Japan, Tell 8I43 235731 9, Fax 81432357319 ,

Email yoshiaki.h.k0523@gmail.com

Received: April 19, 2017| Published: April 27, 2017
Abbreviations: AACN, American association of critical care nurses; I-CVI, item-level content validity index; Japanese synergy model, japanese version of the AACN synergy model for patient care; Synergy model, the AACN synergy model for patient care

\section{Introduction}

The AACN Synergy Model for Patient Care ${ }^{1}$ has attracted attention in recent years as a middle-range theory of nursing practice that focuses on the patient-nurse relationship. The synergy model consists of patient characteristics and nurse competencies. Patient characteristics comprise resiliency, vulnerability, stability, complexity, resource availability, participation in care, participation in decision making, and predictability. Nurse competencies comprise clinical judgment, advocacy, caring practices, collaboration, systems thinking, response to diversity, clinical inquiry, and the facilitation of learning.

Therefore, patient characteristics and nurse competencies each consist of eight items, with each item scored between Level 1 (poor) and Level 5 (good). The synergy model is a middle-range theory that identifies the nursing practices required encouraging autonomy on the part of patients and family members by ascertaining their needs in terms of patient characteristics, and identifying the nursing competencies required to deal with these characteristics.

The synergy model has been shown to improve patient satisfaction and nurses' performance; ${ }^{2}$ additionally, Galton and Brett found that the use of the synergy model enables the clear transmission of information. ${ }^{3}$ Kaplow reported case studies of the use of the synergy model in clinical training, ${ }^{4}$ and Green found that the practical application of the synergy model in training and healthcare improved learning outcomes. ${ }^{5}$ Rozdilsky and Alecxe produced assessments based on patient characteristics, and found that incident rates, nosocomial infections, and falls all declined after these assessments were introduced. ${ }^{6}$ Swickard, Swickard, Reimer, Lindell, and Winkelman carried out assessments of the condition of patients undergoing transport, and produced a triage sheet to determine to assign nurses to care for these patients. ${ }^{7}$ Although the synergy model is already being employed in a variety of research methodologies, no Japanese study using this model has yet been performed. We therefore carried out a preliminary test using the Japanese version of the synergy model, with the aim of ascertaining the effects of the use of the synergy model in Japan.

\section{Materials and methods}

\section{Preparation of the japanese version of the AACN synergy model for patient care}

Work on preparing the Japanese version of the AACN Synergy Model for Patient Care was started after permission had been obtained from the American Association of Critical-Care Nurses (AACN) who developed the original model. The AACN did not take part in the translation work, and is not responsible for the accuracy of the translation. The AACN takes no responsibility for any errors in the translation, nor for any damage that may occur due to the use of the translated version. The original reference and copyright information for the AACN Synergy Model for Patient Care are as follows:

American Association of Critical-Care Nurses. The AACN Synergy Model for Patient Care. Aliso Viejo, CA: American Association of Critical-Care Nurses. 
http://www.aacn.org/wd/certifications/content/synmodel. pcms?pid $=1 \&=\&$ menu $=$ certification. C 2016 by AACN. All rights reserved.

To achieve conceptual equivalence between the Japanese and original versions, the Japanese version was prepared with reference to the Process of translation and adaptation of instruments proposed by the World Health Organization (WHO) ${ }^{8}$

Forward translation: The parallel translation method recommended in many studies ${ }^{9-11}$ was chosen for the forward translation in order to minimize the cultural bias in the terminology used in the original. Three translators produced their own translations independently, and then repeatedly discussed and amended the content of these translations. All of the three translators $(1,2,3)$ are native speakers of Japanese and bilingual in Japanese and English. Translator 1 had conducting research to develop a Japanese version scale and is an ICU staff nurse PhD. Translator 2 had lived in the United States for 1 year, and had worked as a postdoctoral fellow. Translator 3 is the primary investigator.
Back translation and expert panel: Back translation ${ }^{12}$ was performed to verify the consistency of the Japanese version with the original. An expert panel, consisting of four bilingual nursing researchers is health professionals with no previous knowledge of the Synergy model and a professional translator compared the original and the Japanese version (back translation) to check whether any expressions had been inappropriately translated, and corrected the Japanese version to include more appropriate terms. We also investigated whether the content of the Japanese version of the synergy model was appropriate for evaluating nursing practice in Japan. The validity of the translated content was investigated by means of an evaluation of all the items by members of the expert panel using the Item-level Content Validity Index (I-CVI). Content validity was assessed according to the criteria recommended by Polit $\&$ Beck. ${ }^{13}$ The content was graded on a fourpoint scale as $1=$ not relevant, $2=$ somewhat relevant, $3=$ quite relevant, or $4=$ highly relevant, with items graded as 3 or 4 being regarded as valid. The translated content was revised for items graded 1 or 2 . The correction process was repeated until all the researchers who took part in the expert panel reached a consensus agreement on the validity of the items. Figure 1 show the translation process used in this study.

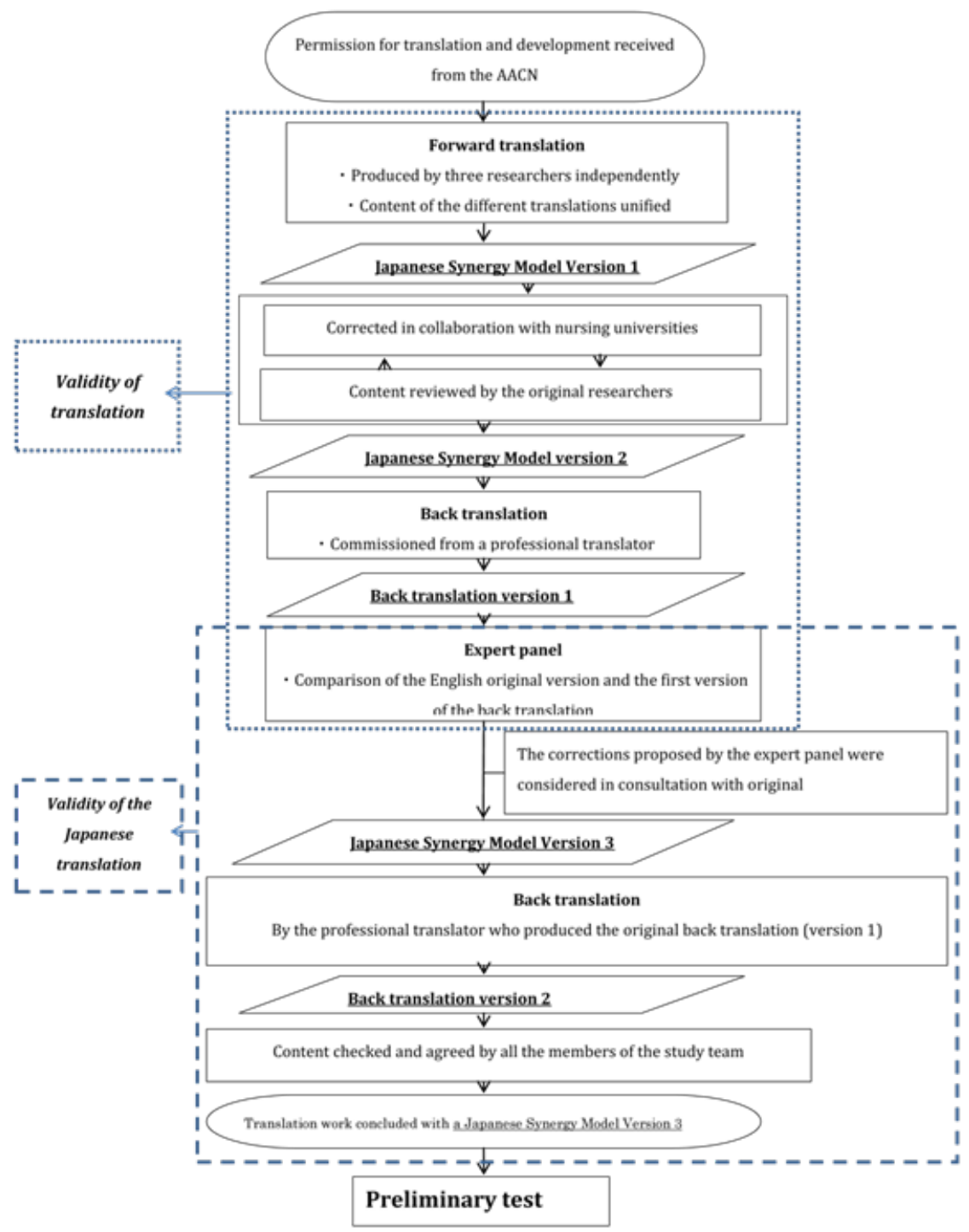

Figure I The translation process used in this study 


\section{Preliminary testing}

A preliminary test was carried out to verify the effect of using the Japanese version of the synergy model. The effect of the synergy model lies in the production of a blueprint for nursing practice. ${ }^{1}$ Its effect on nursing practice can thus be verified by investigating the perspectives of multiple nurses on patient characteristics and the nursing competencies required to treat them. We therefore asked multiple nurses to grade the same case studies with respect to the eight patient characteristics and eight nursing competencies, and investigated the effect of using the Japanese version of the synergy model by identifying trends in nurses' assessments. Preliminary testing was carried out using simulated cases based on two case studies reported overseas. ${ }^{14}$ Case 1 is the patient of acute myocardial infarction. Case 2 is the patient that is considering the possibility of heart transplantation. Both cases were patients with acute stage disease. Permission to use these case studies in the present study was obtained from the AACN, the copyright holder of the material.

Participants: The participants in this study were nurses with more than 5 years of experience, working in critical care wards (Intensive Care Units or High Care Units) at a university hospital. The survey took the form of an anonymous self-administered questionnaire, which the participants were requested to place in a collection box after completion. This study was approved by the Tsukuba University Hospital Clinical Research Ethics Review Board (H28-96). Participants voluntarily participated in this research, and they were informed that they would not suffer any disadvantages if they did not participate, that their questionnaire responses were anonymous, and that their anonymity would be safeguarded

Study content: Respondents were asked to provide their number of years of nursing experience. The items concerning the Japanese version of the synergy model comprised the importance of each of the patient characteristics and nursing competencies for each of the case studies. Respondents were asked to indicate how important they would judge each item to be if they themselves were nursing the case study patients. Responses were given according to a complete ranking method, with the importance of each of the eight patient characteristics and nursing competencies in the case studies ranked in order from 1 (most important) to 8 (least important).

Analysis: Spreadsheet software (Microsoft Excel 2010) and statistical software (SPSS Statistics 22, IBM Corporation) were used for statistical analysis. The importance of the patient characteristics and nursing competencies were investigated using a complete ranking method to rank each of the eight items. The complete ranking method was tested by calculating Kendall's coefficient of concordance for the importance of each patient characteristic and nursing competency. The significance level used in all analyses was .05.

\section{Results and discussion}

\section{Preparation of the japanese version of the AACN synergy model for patient care}

Back translation was performed twice. The first version of the back translation was discussed by the expert panel. Version 3 of the Japanese synergy model was then produced on the basis of the comments made by the panel members. The content of the second version of the back translation was checked by the researchers who took part in the expert panel, with all the items scoring 3 or 4 on the I-CVI. All the members of the study team who had been involved in the preparation of the Japanese version agreed that the task of preparing the Japanese version of the synergy model was complete with Version 3.

\section{Preliminary testing}

Participant characteristics: Participants in this study were 13 nurses with at least five years nursing experience (mean $9.9 \pm 4.5$ years). Of the 13 participants, 11 provided responses with no missing values, and these were used for the analysis.

Importance of patient characteristics and nurse competencies in each case study: Table 1 shows the means and standard deviations for the order of importance of patient characteristics assigned by nurses for both simulated case studies.

Table I The other of importance assigned to participant characteristics in two simulated case studies

\begin{tabular}{|c|c|c|}
\hline Patient characteristics & Case I $(n=I I)$ & Case $2(n=I I)$ \\
\hline Rank I-8 & Mean士SD & Mean士SD \\
\hline Resiliency & $2.4 \pm 1.9$ & $2.1 \pm 1.9$ \\
\hline Vulnerability & $3.5 \pm 2.1$ & $4.9 \pm 1.8$ \\
\hline Stability & $2.8 \pm 0.9$ & $3 \pm 1.5$ \\
\hline Complexity & $4.5 \pm 2.1$ & $4.5 \pm 2.2$ \\
\hline Resource availability & $5.6 \pm 2.1$ & $6.5 \pm 1.4$ \\
\hline Participation in care & $6.2 \pm 1.5$ & $5.9 \pm 2.1$ \\
\hline Participation in decision making & $5.3 \pm 2.3$ & $3.9 \pm 1.8$ \\
\hline Predictability & $5.8 \pm 2$ & $4.8 \pm 2.4$ \\
\hline
\end{tabular}

Table 2 shows the means and standard deviations for the order of importance of nursing competencies assigned by nurses in both case studies.

Table 2 The order of importance assigned to nursing competencies in two simulated case studies

\begin{tabular}{|c|c|c|}
\hline Nurse competencies & Case I $(n=I I)$ & Case $2(n=I I)$ \\
\hline Rank I-8 & Mean \pm SD & Mean \pm SD \\
\hline Clinical judgment & $2.4 \pm 2.5$ & $2.2 \pm 2$ \\
\hline Advocacy and moral agency & $3.5 \pm 2.1$ & $3.8 \pm 1.6$ \\
\hline Caring practices & $2.7 \pm 1.6$ & $2.9 \pm 1.6$ \\
\hline Collaboration & $3.9 \pm 1.7$ & $3.1 \pm 1.3$ \\
\hline Systems thinking & $4.4 \pm 1.9$ & $4.9 \pm 1.7$ \\
\hline Response to diversity & $5.7 \pm 2.1$ & $6.4 \pm 2.1$ \\
\hline Clinical inquiry & $7.1 \pm 1.3$ & $6.3 \pm 1.9$ \\
\hline Facilitation of learning & $5.1 \pm 2.1$ & $6.5 \pm 0.9$ \\
\hline
\end{tabular}

Kendall's coefficient of concordance was calculated to investigate whether the assignment of the order of importance of patient characteristics by the nurses was consistent. This revealed slight but statistically significant consistencies between the importances assigned to patient characteristics by nurses in Cases 1 and 2 (Table $3)$. 
Table 3 Tendency toward consistency in the importance assigned to patient characteristics

\begin{tabular}{lllll}
\hline & Kendall's W & $\chi^{2}$ & df & P value \\
\hline Case I & 0.356 & 27.39 & 7 & $<0.05$ \\
Case 2 & 0.358 & 27.57 & 7 & $<0.05$ \\
\hline
\end{tabular}

Kendall's coefficient of concordance was also calculated to investigate whether the order of importance assigned to nursing competencies was consistent within participants. This analysis revealed significant consistencies in the order of importance assigned to nursing competencies by nurses in both cases, although the consistency in agreement was only slight in Case 1, and moderate in Case 2 (Table 4).

Table 4 Tendency toward consistency in the importance assigned to nursing competencies

\begin{tabular}{lllll}
\hline & Kendall's W & $\chi^{2}$ & df & P value \\
\hline Case I & 0.396 & 27.7 & 7 & $<0.05$ \\
Case 2 & 0.499 & 38.42 & 7 & $<0.05$ \\
\hline
\end{tabular}

\section{Discussion}

Participants and case studies: The synergy model is used in a variety of different areas, and in most cases the participants are adults in the field of critical care. We therefore selected nurses working in wards treating adults requiring critical care as study participants. The two case studies used in our research are cases that have been described in previous studies. ${ }^{14}$ By selecting case studies with comprehensive descriptions, we chose valid scenarios for explaining the synergy model and achieving the study objectives. However, previous studies have identified the type of information required for nurses to evaluate patients, ${ }^{15}$ and the information provided in the case studies used in our study may have been insufficient for evaluation by comparison. The resulting difficulty in assessing patient characteristics may have affected the assessment of the nursing competencies required in their treatment.

\section{Preparation of the Japanese version of the AACN synergy model for patient care}

There are various published guidelines and procedures for preparing translations. ${ }^{8,9,16,17}$ Many previous studies have indicated that translations should be prepared by the use of back translations and an expert panel. Several bilingual nursing researchers participated in the study from the preparation of the forward translation and scrutinized the content of the translation. The back translation process also involved a professional translator, who possessed the abilities required for our study. The researchers who took part in the expert panel evaluated the validity of the content of the Japanese version of the synergy model using a content validity index, and scrutinized the translated model until it fulfilled the validity criteria recommended in previous studies.

The preparation of a translated instrument must ensure not only the validity of the content but also conceptual equivalence between the original and the final translation. "Conceptual equivalence" refers to whether the concepts expressed in the language used in the original still carry their original meaning after they have been translated. ${ }^{18}$ The AACN, which holds the copyright to the synergy model, required that the people who produced the original version should not be requested to compare the back translation with the original. Therefore, the authors of the original version did not confirm the equivalence between versions. However, several researchers collaborated on the forward translation and scrutinized the content meaning in the expert panel by comparing the back translation with the original, correcting it several times in order to produce the Japanese version. Although we are unable to conclude that the Japanese version of the synergy model produced in this study is conceptually equivalent to the original, the translation was completed by the most appropriate translation process in an attempt to achieve this.

\section{Use of the Japanese version of the synergy model}

As only two case studies were used for evaluation in this study, this was too few for us to investigate inter-rater reliability (an index of reliability). Future studies involving more participants and patients are required in order to investigate reliability in this way.

The outcome indicators of the synergy model are patient and family satisfaction, rate of adverse incidents, complication rate, adherence to the discharge plan, mortality rate, and patient's length of stay. ${ }^{19}$ In this study, because we used case studies for our investigation, we did not verify the effect on these outcome indicators. The method of investigating the assigned order of importance of patient characteristics and nursing competencies that we used in this study has not previously been used. We found that there was significant, although slight, consistency in the order of importance assigned to the patient characteristics and nursing competencies in both case studies, suggesting that the use of the Japanese version of the synergy model may provide the same effects provided by the original. However, the limited number of participants in our study meant that we were unable to perform comparisons in terms of years of experience or the type of ward on which they worked. Brewer et al. ${ }^{20}$ reported that assessments vary depending on the level of specialist knowledge of the synergy model. Becker et al..$^{21}$ also found that awareness of the importance of nursing competencies varied between critical care nurse practitioners and critical care specialist nurses. Further research is required to investigate whether individual factors, such as qualifications held and professional role, have any effect on nurses' judgments.

\section{Conclusion}

In this study, we developed a Japanese version of the synergy model and ran preliminary tests to establish whether our translated version could be used in future to measure the synergy between patient characteristics and the provision of nursing competencies.

We produced a Japanese version of the synergy model and used this to carry out a preliminary test, from which we drew the following conclusions. We found that the assignment of importance to each of the items in the Japanese version was statistically significant, suggesting that the same effects may be obtained from the use of the Japanese version of the synergy model as from the use of the original. There were some limitations to the description of the effect of the Japanese version of the synergy model in this study. Further studies involving as many participants as possible are therefore required in order to statically verify the reliability and validity of the Japanese version of the synergy model, with a view to enabling its practical use.

\section{Acknowledgements}

This study was funded in part by a Grant-in-Aid for Scientific Research A (No. 16H02693) from the Japanese Ministry of Education, Culture, Sports, Science and Technology. 


\section{Conflicts of interest}

The authors have no conflicts of interest directly relevant to the content of this article.

\section{References}

1. Curley MA. Patient-nurse synergy: Optimizing patients outcomes. Am J Crit Care. 1998;7(1):64-72.

2. Khalifehzadeh A, Jahromi MK, Yazdannik A. The impact of synergy model on nurses' performance and the satisfaction of patients with acute coronary syndrome. Iran J Nurs Midwifery Res. 2012;7(1):16-20.

3. Gralton K, Brett S. Integrating the synergy model for patient care at children's hospital of Wisconsin. J Pediatr Nurs. 2021;27(1):74-81.

4. Kaplow R. Applying the synergy model to nursing education. Crit Care Nurses. 2002;22(3):77-81.

5. Green DA. A synergy model of nursing education. J Nurses Staff Dev. 2006;22(6):277-283

6. Rozdilsky J, Alecxe A. Saskatchewan: Improving patient, nursing and organizational outcomes utilizing formal nurse-patient ratios. Nurs Leadersh (Tor Ont). 2012;25:103-13.

7. Swickard S, Swickard W, Reimer A, et al. Adaptation of the AACN synergy model for patient care to critical care transport. Crit Care Nurse. 2014;34(1):16-28.

8. World health organization (n.d.) Process of translation and adaptation of instruments.

9. Harkness J. Questionnaire Translation 1st ed. In: Harkness J, Fons JR Van De Vijver, et al. editors. Cross-cultural Survey Methods. New jersey, USA: Springer; 2003. p. 35-56.

10. Peters M, Passchier J. Translating instruments for Cross-Cultural studies in headache research. Headache. 2006;46(1):82-91.
11. Douglas SP, Craig CS. Collaborative and iterative translation: An alternative approach to back translation. Journal of International Marketing. 2007;15(1):30-43.

12. Brislin RW. Back-translation for cross-cultural research. J Cross-Cult Psychol. 1970;1(3):185-216.

13. Polit D, Beck C. The content validity index: Are you sure you know what's being reported? Critique and recommendations. Res Nurs Health. 2006;29(5):489-497.

14. Edwards DF. The synergy model: Linking patient needs to nurse competencies. Crit Care Nurse. 1999;19(1):88-90.

15. Kohr L, Hickey P, Curley M. Building a nursing productivity measure based on the synergy model: First steps. Am J Crit Care. 2012;21(6):420 430.

16. International test commission. ITC guidelines for translating and adapting tests; 2005.

17. American council of learned societies. Social science translation project; 2006.

18. Behling O, Law KS. Translating questionnaires and other research instruments: Problems and solutions. USA: Sage Publications; 2000.

19. Hardin SR. Introduction to the AACN synergy model for patient care. In: SR Hardin, R Kaplow editors. Synergy for Clinical Excellence: The AACN Synergy Model for Patient Care. USA: Jones and Bartlett Publishers; 2005. p. 3-10.

20. Brewer BB, Wojner Alexandrov AW, Triol N, et al. AACN synergy model's characteristics of patients: Psychometric analyses in a tertiary care health system. Am J Crit Care. 2007;16(2):158-167.

21. Becker D, Kaplow R, Muenzen P, et al. Activities performed by acute and critical care advanced practice nurses: American association of criticalcare nurses study of practice. Am J Crit Care. 2006;15(2):30-148. 